\title{
The role of physical activity in metabolic homeostasis before and after the onset of type 2 diabetes: an IMI DIRECT study
}

\author{
Robert W. Koivula ${ }^{1,2}$ (D) Naeimeh Atabaki-Pasdar ${ }^{1} \cdot$ Giuseppe N. Giordano $^{1} \cdot$ Tom White $^{3} \cdot$ Jerzy Adamski $^{4,5,6}$. \\ Jimmy D. Bell ${ }^{7}$ • Joline Beulens ${ }^{8}$ - Søren Brage ${ }^{3,9} \cdot$ Søren Brunak ${ }^{10,11}$ • Federico De Masi ${ }^{10,11}$. \\ Emmanouil T. Dermitzakis ${ }^{12,13,14} \cdot$ Ian M. Forgie ${ }^{15} \cdot$ Gary Frost $^{16} \cdot$ Torben Hansen $^{9,17} \cdot$ Tue H. Hansen ${ }^{17}$. \\ Andrew Hattersley ${ }^{18,19}$. Tarja Kokkola ${ }^{20}$ - Azra Kurbasic ${ }^{1}$ - Markku Laakso ${ }^{20}$ - Andrea Mari ${ }^{21} \cdot$ Timothy J. McDonald $^{18}$. \\ Oluf Pedersen ${ }^{17}$. Femke Rutters ${ }^{8}$ - Jochen M. Schwenk ${ }^{22}$ - Harriet J. A. Teare ${ }^{23}$. E. Louise Thomas ${ }^{7}$. \\ Ana Vinuela ${ }^{12,13,14}$ - Anubha Mahajan ${ }^{24}$ - Mark I. McCarthy 2,24,25,26 Hartmut Ruetten $^{27}$ - Mark Walker $^{28}$. \\ Ewan Pearson $^{15} \cdot$ Imre Pavo $^{29} \cdot$ Paul W. Franks $^{1,2,30,31} \cdot$ for the IMI DIRECT Consortium
}

Received: 29 August 2019 / Accepted: 29 November 2019/Published online: 30 January 2020

(C) The Author(s) 2020

\begin{abstract}
Aims/hypothesis It is well established that physical activity, abdominal ectopic fat and glycaemic regulation are related but the underlying structure of these relationships is unclear. The previously proposed twin-cycle hypothesis (TC) provides a mechanistic basis for impairment in glycaemic control through the interactions of substrate availability, substrate metabolism and abdominal ectopic fat accumulation. Here, we hypothesise that the effect of physical activity in glucose regulation is mediated by the twincycle. We aimed to examine this notion in the Innovative Medicines Initiative Diabetes Research on Patient Stratification (IMI DIRECT) Consortium cohorts comprised of participants with normal or impaired glucose regulation (cohort $1: N \leq 920)$ or with recently diagnosed type 2 diabetes (cohort 2: $N \leq 435$ ).

Methods We defined a structural equation model that describes the TC and fitted this within the IMI DIRECT dataset. A second model, twin-cycle plus physical activity (TC-PA), to assess the extent to which the effects of physical activity in glycaemic regulation are mediated by components in the twin-cycle, was also fitted. Beta cell function, insulin sensitivity and glycaemic control were modelled from frequently sampled $75 \mathrm{~g}$ OGTTs (fsOGTTs) and mixed-meal tolerance tests (MMTTs) in participants without and with diabetes, respectively. Abdominal fat distribution was assessed using MRI, and physical activity through wristworn triaxial accelerometry. Results are presented as standardised beta coefficients, SE and $p$ values, respectively.

Results The TC and TC-PA models showed better fit than null models (TC: $\chi^{2}=242, p=0.004$ and $\chi^{2}=63, p=0.001$ in cohort 1 and 2, respectively; TC-PA: $\chi^{2}=180, p=0.041$ and $\chi^{2}=60, p=0.008$ in cohort 1 and 2 , respectively). The association of physical activity with glycaemic control was primarily mediated by variables in the liver fat cycle.

Conclusions/interpretation These analyses partially support the mechanisms proposed in the twin-cycle model and highlight mechanistic pathways through which insulin sensitivity and liver fat mediate the association between physical activity and glycaemic control.
\end{abstract}

Keywords Beta cell function $\cdot$ Ectopic fat $\cdot$ Glycaemic control $\cdot$ Insulin sensitivity $\cdot$ Physical activity $\cdot$ Prediabetes $\cdot$ Structural equation modelling · Type 2 diabetes

Electronic supplementary material The online version of this article (https://doi.org/10.1007/s00125-019-05083-6) contains peer-reviewed but unedited supplementary material, which is available to authorised users.

Robert W. Koivula

robert.koivula@med.lu.se

Extended author information available on the last page of the article

\begin{tabular}{|c|c|}
\hline \multicolumn{2}{|l|}{ Abbreviations } \\
\hline CFI & Comparative fit index \\
\hline DiRECT & $\begin{array}{l}\text { Primary care-led weight management } \\
\text { for remission of type } 2 \text { diabetes trial }\end{array}$ \\
\hline fsOGTT & Frequently sampled OGTT \\
\hline hpfVM & High-pass filtered vector magnitude \\
\hline IMI DIRECT & Innovative Medicines Initiative \\
\hline & Diabetes Research on Patient Stratification \\
\hline MMTT & Mixed-meal tolerance test \\
\hline
\end{tabular}




\section{Research in context}

\section{What is already known about this subject?}

- Physical activity, abdominal ectopic fat and glycaemic regulation are related

- The previously proposed twin-cycle hypothesis provides a mechanistic basis for impairment in glycaemic control through the interactions of substrate availability, substrate metabolism and abdominal ectopic fat accumulation

What is the key question?

- Is the positive effect of physical activity on glycaemic regulation mediated by features of the twin-cycle?

What are the new findings?

- Model-fit statistics of structural equation models based on the twin-cycle hypothesis fitted on two cohorts (with recently diagnosed diabetes and without diabetes) within IMI DIRECT indicate good model fit

- Individual effect estimates indicate stronger support for relationships in the liver fat cycle compared with those in the pancreatic fat cycle

- Insulin sensitivity and ectopic liver fat mediated the association of physical activity with glycaemic regulation

How might this impact on clinical practice in the foreseeable future?

- Physical activity, as a modifiable behaviour, has the potential to be a target for interventions seeking to improve glycaemic regulation through improving peripheral insulin sensitivity and reducing ectopic liver fat

$\begin{array}{ll}\text { NAFLD } & \text { Non-alcoholic fatty liver disease } \\ \text { OGIS } & \text { Oral glucose insulin sensitivity } \\ \text { RMSEA } & \text { Root mean square error of approximation } \\ \text { TC } & \text { Twin-cycle hypothesis } \\ \text { TC-PA } & \text { Twin-cycle plus physical activity } \\ \text { TLI } & \text { Tucker-Lewis index }\end{array}$

\section{Introduction}

The global epidemics of type 2 diabetes and obesity $[1,2]$ follow in the wake of rapid urbanisation, reduced physical activity, and ageing populations [3]. Physical inactivity is strongly associated with peripheral insulin resistance, abdominal obesity and glucose dysregulation [4-7]. Physical inactivity may also predispose to non-alcoholic fatty liver disease (NAFLD) [8], which in turn may also adversely affect glucose homeostasis [9-11]. Indeed, recent Mendelian randomisation studies hint at bidirectional causal relationships between NAFLD and type 2 diabetes $[12,13]$. Various mechanisms for these relationships have been proposed [14], with chronic positive energy balance considered a primordial modifiable risk factor $[15,16]$. This notion is articulated through the twin-cycle model, whereby the first cycle describes liver fat accumulation leading to reduced suppression of hepatic gluconeogenesis, consequential elevations in both fasting glucose and insulin concentrations, and hepatic lipid production; the second cycle focuses on the pancreas, where elevated circulating lipids accumulate in the pancreas, impairing endogenous insulin secretion $[15,16]$. However, the extent to which physical activity affects blood glucose homeostasis through mechanisms outlined in the twin-cycle model is unknown.

A better understanding of this would not only add to the physiological understanding of diabetes but might also help guide the design of clinical trials seeking to study the pathogenesis of type 2 diabetes. For example, as physical activity is a modifiable behaviour, it is a potential target for interventions seeking to modify processes involved in the relationships between NAFLD and type 2 diabetes. Furthermore, studies seeking to assess these relationships would benefit from understanding which factors are affected by physical activity and mediate the effect of physical activity in glycaemic control.

Multivariate structural analyses (such as structural equation modelling) can be a powerful way to address these putative effects but require accurate and concurrent assessments of glycaemic control, abdominal fat distribution and lifestyle variables in adequately sized cohorts, few of which currently exist. The Innovative Medicines Initiative Diabetes Research on Patient Stratification (IMI DIRECT) cohorts [17, 18] are well-suited for such analyses.

The purpose of this study was to test potential mechanisms mediating the effects of physical activity in glycaemic control before and after the onset of type 2 diabetes.

\section{Methods}

\section{Study cohorts}

These analyses were conducted in two parallel cross-sectional cohorts of European ancestry adults from northern Europe: the 
first cohort (cohort 1) comprised of participants with blood glucose concentrations within the normal glucose control or prediabetes (impaired $\mathrm{HbA}_{1 \mathrm{c}}$, fasting glucose or $2 \mathrm{~h}$ glucose according to ADA criteria [19]) brackets and the second cohort (cohort 2) comprised individuals with recently diagnosed type 2 diabetes (within 6-36 months of study enrolment). Participants underwent detailed physical examinations, including MRI scans and carbohydrate challenge tests, diet assessment and objective habitual physical activity assessment. Approval for the study protocol was obtained from each of the regional research ethics review boards separately and all participants provided written informed consent at enrolment. The research conformed to the ethical principles for medical research involving human participants outlined in the declaration of Helsinki.

The study rationale and design and core characteristics of the IMI DIRECT cohorts are reported in detail elsewhere [17, 18]. Below, we provide a summary and describe the methods most relevant for the present analyses.

Cohort 1 (prediabetes) was from a sampling frame of 24,196 participants nested within prospective cohorts from Denmark (Copenhagen), Finland (Kuopio), the Netherlands (Hoorn) and Sweden (Malmö); 2127 participants at varying risk of glycaemic deterioration were enrolled into the study. To determine the risk of rapid glycaemic deterioration, we used the DIRECT-DETECT algorithm [20]. For cohort 2 (diabetes), 789 participants were recruited from health registries and primary care practices in Denmark (Copenhagen), the UK (Dundee, Exeter, Newcastle), the Netherlands (Hoorn) and Sweden (Lund). As neither of the Swedish study centres undertook MRI scans, they were not included in the current analysis.

Of these participants, 920 (cohort 1) and 435 (cohort 2) had all the necessary variables for a complete case analysis of the twin-cycle hypothesis (TC) model and 725 (cohort 1) and 361 (cohort 2) had all the necessary variables for the complete case analyses fitting the twincycle plus physical activity (TC-PA) model. The following variables were included in these models: fasting plasma glucose, $2 \mathrm{~h}$ glucose, oral glucose insulin sensitivity (OGIS), liver fat, pancreatic fat, fasting insulin secretion rate, glucose sensitivity (insulin secretion per glucose), age, sex, centre, metformin use, total daily energy intake, total daily carbohydrate, protein and fat intake, and mean daily physical activity intensity. The characteristics of these subcohorts are shown in Table 1.

\section{Measures}

Fasting glucose was assessed from venous plasma samples drawn in the morning following an overnight fast. Frequently sampled $75 \mathrm{~g}$ oral glucose tolerance tests (fsOGTTs) and mixed-meal tolerance tests (MMTTs) were carried out in cohort 1 and 2, respectively. Mixed meals (250 $\mathrm{ml}$ Fortisip liquid drink [18.4 g carbohydrate per $100 \mathrm{ml}$ ) rather than $75 \mathrm{~g}$ oral glucose loads were used in cohort 2 to minimise the risk of severe hyperglycaemia as participants had type 2 diabetes. The $2 \mathrm{~h}$ glucose, OGIS, fasting insulin secretion rate and glucose sensitivity (dose-response slope of insulin secretion in response to glucose) were calculated from the fsOGTT and MMTT data, as described elsewhere [21, 22]. Liver and pancreatic fat were measured by MRI and quantified using a multiecho technique described in detail elsewhere $[17,18,23$, 24]. Briefly, prone $1.5 \mathrm{~T}$ to $3 \mathrm{~T}$ images (depending on availability at each study centre) were acquired. T1weighted images were obtained for the abdominal region (between the diaphragm and acetabulum) with maximum field of view and $10 \mathrm{~mm}$ slice thickness with a $10 \mathrm{~mm}$ slice gap. A three-dimensional scan using 50-80 images at slice thicknesses of 1.2-2 $\mathrm{mm}$ (depending on equipment) was acquired to image the pancreas. Further axial singleslice multi-echo images were acquired of the liver and pancreas (10 $\mathrm{mm}$ slice thickness). Whole-organ pancreatic and liver fat estimates were then inferred from these images, where experienced radiographers manually determined organ boundaries. Physical activity was objectively assessed using triaxial accelerometry (ActiGraph GT3X+, ActiGraph, Pensacola, FL, USA) on the non-dominant wrist over 10 days. Physical activity intensity was characterised by calculating the mean high-pass filtered vector magnitude (hpfVM) of the triaxial acceleration signal $[25,26]$; the mean of this was used to describe overall physical activity level. Non-wear was inferred as a vector magnitude SD of less than $4 \mathrm{mg}$ for a consecutive period greater than $60 \mathrm{~min}$. To account for bias introduced by removal of non-wear time in combination with differential diurnal non-wear patterns between individuals, adjustments were made for diurnal rhythm [27]. Dietary intake was assessed using a validated multi-pass food habit questionnaire and $24 \mathrm{~h}$ diet record, as previously described [17, 18].

\section{Statistical analysis}

All continuous variables were standardised by rank-normal transformed (mean 0, SD 1) by sex (and by lifestyle vs metformin + lifestyle in cohort 2). Adjustment for putative confounders was done by two-step residual regression where, in the first step, residuals were extracted from general linear models undertaken on the transformed continuous variables or binary categorical variables, and these residuals were used in subsequent models as either outcome or predictor variables. Regression models for residual extraction co-varied for age, study centre, total daily energy intake and total daily intake of dietary 
Table 1 Characteristics of cohort subset used in each model

\begin{tabular}{|c|c|c|c|c|}
\hline \multirow[t]{2}{*}{ Characteristic } & \multicolumn{2}{|c|}{$\begin{array}{l}\text { Cohort } 1 \\
\text { (no diabetes/prediabetes) }\end{array}$} & \multicolumn{2}{|c|}{ Cohort 2 (diabetes) } \\
\hline & $\mathrm{TC}$ & TC-PA & $\mathrm{TC}$ & TC-PA \\
\hline Male sex, $\%$ & 83 & 83 & 57 & 60 \\
\hline Age, years & $60.6(6.3)$ & $60.6(6.3)$ & $61.5(8.3)$ & $61.7(8.4)$ \\
\hline BMI, $\mathrm{kg} / \mathrm{m}^{2}$ & $27.8(3.6)$ & $27.8(3.7)$ & $30.5(4.8)$ & $30.4(4.6)$ \\
\hline Fasting glucose, mmol/1 & $5.8(0.5)$ & $5.8(0.5)$ & $7(1.5)$ & $7(1.4)$ \\
\hline $2 \mathrm{~h}$ glucose, $\mathrm{mmol} / \mathrm{l}$ & $6(1.7)$ & $6(1.7)$ & $8.6(2.9)$ & $8.5(2.8)$ \\
\hline Fasting triacylglycerol, mmol/l & $1.4(0.6)$ & $1.4(0.7)$ & $1.5(0.9)$ & $1.5(0.9)$ \\
\hline Fasting insulin, pmol/1 & $73(49)$ & $75(51)$ & $105(69)$ & $105(68)$ \\
\hline Fasting insulin secretion, pmol $\mathrm{min}^{-1} \mathrm{~m}^{-2}$ & $105(40)$ & $106(41)$ & $134(49)$ & $136(50)$ \\
\hline Glucose sensitivity, pmol $\min ^{-1} \mathrm{~m}^{-2} \mathrm{mmol} \mathrm{l}^{-1}$ & $107(50)$ & $107(50)$ & $85(54)$ & $89(56)$ \\
\hline 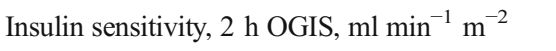 & $374(56)$ & $374(56)$ & $302(71)$ & $302(70)$ \\
\hline Liver fat, \% & $5(4.7)$ & $5(4.7)$ & $8.6(7.2)$ & $8.9(7.4)$ \\
\hline Pancreatic fat, $\%$ & $13.5(9)$ & $13.6(9.1)$ & $11.2(7.2)$ & $11.7(6.9)$ \\
\hline
\end{tabular}

Values are mean (SD), except for male sex, which is \% of subcohort carbohydrates, fats and proteins. Pearson correlation coefficients were generated and plotted in a matrix to illustrate simple pairwise relationships between all model variables.

\section{Structural equation modelling}

We used structural equation modelling to test the overall model fit and relationships between sets of variables within the hypothesised twin-cycle. Structural equation modelling is a multivariate statistical method that can be thought of as a combination of regression analysis, factor analysis and pathway analysis. In a structural equation model a structure (a pathway network) of relationships between variables can be defined according to a prespecified hypothesis (such as the twin-cycle). Based on the observed covariance between the variables in the model, the fit of the defined model can then be tested. In addition to this, pathway (mediation) effects within the defined model can also be tested. We defined models using measured variables only (manifest nodes) and fitted under a maximum likelihood framework using covariance matrices. The model definition (see Figs 1a, 2a) reflects the hypothesised twin-cycle model, proposed elsewhere $[15,16]$. Here, relationships (edge estimates) between variables are adjusted for putative confounders (through two-step residual regression, described above, hence fitted on covariance matrices of the extracted residuals). Direct (non-mediated) edge estimates also account for covariance of the other edges pointing to the same outcome. In other words, edges (see arrows in Figs 1,2) in the model represent regression coefficients, which co-vary with edges from other variables pointing to the same outcome node (see Text box for node and edge abbreviations). Pathway (indirect) effects were estimated for mediated associations of physical activity with glycaemic control using the coefficient product method [28], where mediation is defined using the approach described by Baron and Kenny [29]. Pathways were tested where statistically significant direct associations (individual, non-mediated, edge estimates) along the whole pathway were also observed. Relative model fit was assessed using the comparative fit index (CFI) and the Tucker-Lewis index (TLI), with values ranging from 0 (no fit) to 1 (perfect fit) [30]; a model with a 'good' fit typically requires both indices to exceed 0.95 [31, 32]. Absolute fit was assessed using root mean square error of approximation (RMSEA). This ranges from 0 to 1 , with 0 indicating a perfect fit [30]. A poorly fitting model is typically defined by RMSEA $>0.06$ [33, 34]. CFI, TLI and RMSEA were not used to formally determine adequacy of fit, as their use in this context is controversial and there is limited consensus on appropriate cut-off values because each index is affected differently by degrees of freedom, model complexity and sample size; it is, however, standard practice to report these along with the $\chi^{2}$. To overcome this, we formally tested model fit by comparing the $\chi^{2}$ of the tested model with $\chi^{2}$ values obtained from variable-randomised null models with identical structures (in other words, the variables were randomly assigned to other nodes in the same structural equation model definition) and applied to the respective covariance matrix used for the tested model. This process was iterated 10,000 times. To determine whether the tested model $\chi^{2}$ values were lower (fitted better) than mean $\chi^{2}$ values, one-sample $t$ tests were used. We also calculated the empirical probability of the $\chi^{2}$ value from the null model being lower than the $\chi^{2}$ value from the tested model by expressing the tested model $\chi^{2}$ value as a quantile within the iterated $\chi^{2}$ values. 
Nodes (upper case) and edges (lower case)

\author{
FG/fg Fasting glucose \\ $\mathrm{Fl} / \mathrm{fi} \quad$ Fasting insulin secretion rate \\ GS/gs Glucose sensitivity (insulin secretion per \\ glucose) \\ IS/is Oral glucose insulin sensitivity \\ LF/lf Liver fat \\ $\mathrm{PA} /$ pa Physical activity \\ PF/pf Pancreatic fat \\ $\mathrm{PG} / \mathrm{pg} 2 \mathrm{~h}$ glucose \\ $\mathrm{TG} / \mathrm{tg} \quad$ Fasting triacylglycerol
}

Multiple testing adjustments were not undertaken, as this analysis sought to validate a single previously hypothesised model where direct effect estimates were nested within this single model, reflecting a single overarching hypothesis. Moreover, where results are consistent across the two subcohorts used here, one might regard this as replication. However, the absence of replication may reflect real differences in diseased and nondiseased states, as opposed to providing evidence of type 1 error.

All statistics were computed using $R$ version 3.5.0 [35]. Structural equation models were fitted using lavaan version 0.6-5 [36]. Models were plotted using semPlot version 1.1.2 (CRAN repository or https://github.com/SachaEpskamp/ semPlot, accessed 20 August 2019). The IMI DIRECT data release version used for these analyses was 'direct 29-03-2019'.

\section{Results}

\section{Pairwise correlations}

Statistically significant pairwise correlations between most of the index metabolic outcomes and physical activity were observed, thereby justifying the main structural equation model analysis to test the underlying structure of these correlations. An overview of the pairwise correlations is presented in electronic supplementary material (ESM) Fig. 1. Of note, in both cohorts, indices of reduced insulin resistance such as the increase of OGIS and the suppression of fasting insulin secretion rate correlated strongly with physical activity. However, pairwise associations between pancreatic fat and glucose sensitivity or fasting glucose were not observed, despite the presence of associations between pancreatic fat and a number of other index metabolic variables in cohort 1 .

\section{Structural equation model}

Structural equation model results are shown in Figs 1 and 2 for TC and TC-PA models, respectively. Effect estimates are presented in Table 2 for direct effects for TC and TC-PA models. Indirect (mediation/pathway) effect estimates of physical activity in fasting glucose and $2 \mathrm{~h}$ glucose variation from the TC-PA model are presented in Table 3 . In the model definition diagrams in Figs 1a and 2a, we illustrate which direct effects (edges, depicted as arrows) were modelled within the structural equation model. We also illustrate the underlying liver cycle and pancreatic cycle within the TC to orientate the reader to the original hypothesis [16]. Below, we first describe the overall model fit and key direct (individual edges) effect estimates between physical activity and the index metabolic outcomes within the model. We then describe indirect (pathway) effect estimates between physical activity and glycaemic regulation mediated by twin-cycle variables.

Model fit The TC model showed better fit than the mean fit of the respective null model in cohort $1\left(\chi^{2}=242\right.$ vs $1005, p<5 \times$ $10^{-10}$ ) and cohort $2\left(\chi^{2}=63\right.$ vs 587, $\left.p<5 \times 10^{-10}\right)$ (see Figs $1 b$, $2 b$.). The fit from a randomised null model (10,000 iterations) was unlikely to be better than the TC model in cohort 1 (empirical $p=0.004$ ) and cohort 2 (empirical $p=0.001$ ). The TC-PA model also showed better fit than the mean fit of the respective null model in cohort $1\left(\chi^{2}=180\right.$ vs $\left.605, p<5 \times 10^{-10}\right)$ and cohort $2\left(\chi^{2}=60\right.$ vs $\left.369, p<5 \times 10^{-10}\right)$. The fit from a randomised null model was unlikely to be better than the TC model in cohort 1 (empirical $p=0.041$ ) and cohort 2 (empirical $p=0.008)$.

Direct (non-mediated) effects Most direct effects estimates in the TC model were statistically significant and were in a direction consistent with this hypothesis in both cohorts (Table 2). One notable exception was the relationship between pancreatic fat and glucose sensitivity, which was not statistically significant in either cohort. Physical activity bore a direct positive association with insulin sensitivity in both cohorts. An inverse direct association between physical activity and fasting insulin secretion was also observed in both cohorts. Physical activity was not directly associated with liver fat or $2 \mathrm{~h}$ glucose in either cohort. An inverse direct association between physical activity and fasting triacylglycerol was observed in both cohorts. Physical activity was inversely associated with glucose sensitivity in cohort 2 only.

Indirect (pathway/mediation) effects The association of physical activity with glycaemic control was primarily mediated by variables in the liver fat cycle. Physical activity was associated with fasting glucose through pathway $\mathrm{PA} \rightarrow \mathrm{IS} \uparrow \rightarrow \mathrm{FG} \downarrow$ in both cohorts and through pathways $\mathrm{PA} \rightarrow \mathrm{FI} \downarrow \rightarrow \mathrm{LF} \downarrow \rightarrow \mathrm{IS} \uparrow \rightarrow \mathrm{FG} \downarrow$, $\mathrm{PA} \rightarrow \mathrm{IS} \uparrow \rightarrow \mathrm{GS} \downarrow \rightarrow \mathrm{FG} \uparrow$, in cohort 1 . Consistently, physical 
a

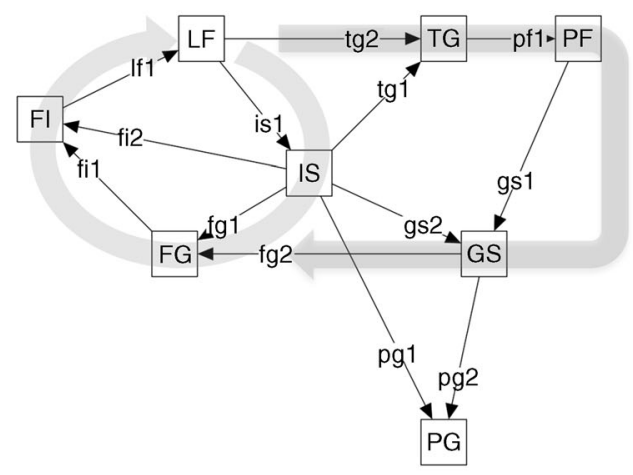

C

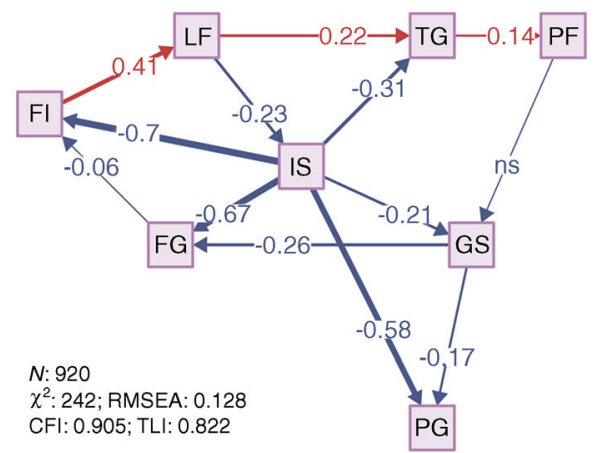

Fig. 1 TC structural equation model definition diagram, fit estimates and effect estimate diagrams from a hypothesised model for the role of physical activity and liver fat in glycaemic control. (a) Model definitions, with squares representing manifest nodes and arrows indicating regression coefficients pointing towards an outcome of a respective regression. (b) Model fit; density plot of model fit $\chi^{2}$ from variable-randomised comparable structural equation models applied on respective dataset $\left(10,000\right.$ iterations). Dashed vertical lines indicate TC model $\chi^{2}$, solid lines

activity was associated with $2 \mathrm{~h}$ glucose through pathway $\mathrm{PA} \rightarrow \mathrm{IS} \uparrow \rightarrow \mathrm{PG} \downarrow$ in both cohorts and through pathway $\mathrm{PA} \rightarrow$ $\mathrm{FI} \downarrow \rightarrow \mathrm{LF} \downarrow \rightarrow \mathrm{IS} \uparrow \rightarrow \mathrm{PG} \downarrow, \mathrm{PA} \rightarrow \mathrm{IS} \uparrow \rightarrow \mathrm{GS} \downarrow \rightarrow \mathrm{PG} \uparrow$ in cohort 1. However, physical activity was associated with both increased fasting glucose and $2 \mathrm{~h}$ glucose through pathways $\mathrm{PA} \rightarrow$ $\mathrm{GS} \downarrow \rightarrow \mathrm{FG} \uparrow$ and $\mathrm{PA} \rightarrow \mathrm{GS} \downarrow \rightarrow \mathrm{PG} \uparrow$ in cohort 2 only. See Table 3 for effect estimates and pathway details and Text box for node abbreviations.

\section{Discussion}

Using structural equations to fit models describing the TC $[15$, 16], we found that in adults with prediabetes and type 2 diabetes overall physical activity volume is associated with multiple metabolic and abdominal ectopic fat features. We also demonstrate that reduced whole-body insulin sensitivity and fasting insulin secretion rate mediate the effects of physical activity in glucose and liver fat homeostasis. This is to our knowledge

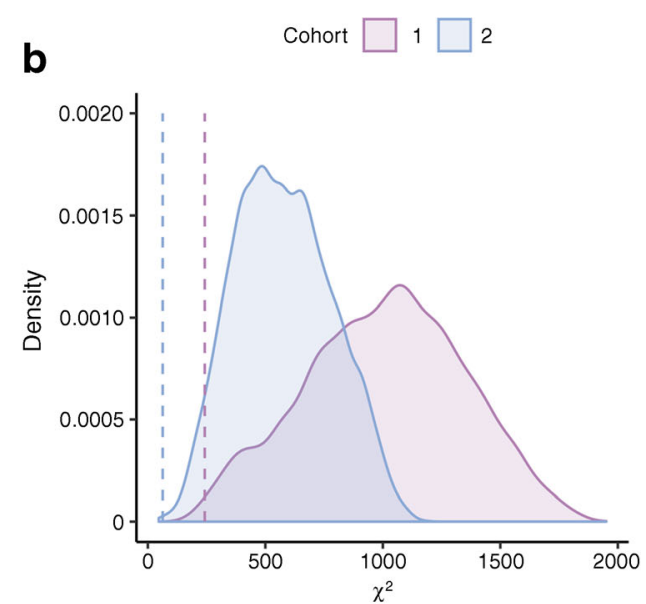

d

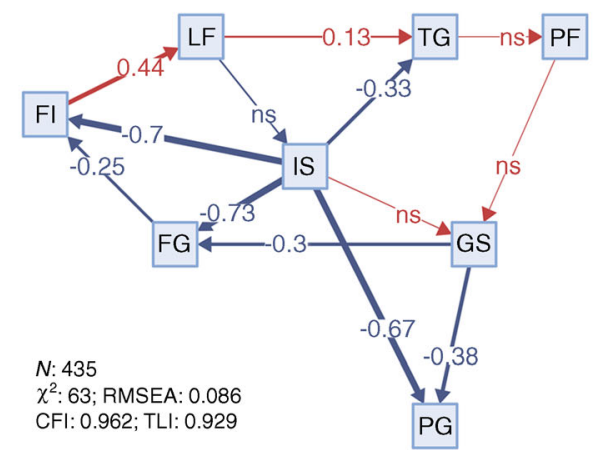

and shaded areas indicate $\chi^{2}$ of all null iterations. (c, d) Effect estimate diagrams of the defined model applied on cohort 1 (no diabetes/prediabetes, c) and cohort 2 (type 2 diabetes, $\mathbf{d}$ ), where the arrow thickness is weighted by effect estimate magnitude, and colours red and blue indicate positive and negative estimates, respectively. All continuous variables are normally transformed and adjusted for age, sex, metformin treatment (cohort 2), study centre, total energy intake, and carbohydrate, fat and protein intake. See Text box for node and edge abbreviations

the first detailed pathway analysis of physical activity and glycaemic regulation.

Our findings partially support the validity of the TC proposed by Taylor $[15,16]$. However, our data do not support the hypothesised effect of pancreatic fat in beta cell function, despite finding that pancreatic fat is associated with numerous other metabolic features; notably, these associations were observed both in conventional pairwise analyses (ESM Fig. 1) and within structural equation models (Figs 1,2 and Table 2). This is important, as these positive results mitigate the possibility that the absence of associations between pancreatic fat and beta cell function is due to measurement error. A key benefit of the approach used here is that the magnitude of the effects of physical activity on glycaemic control is quantified (Table 3), so is likely to prove useful for those planning interventions related to this topic.

The direct effects observed here between liver fat accumulation and reduced insulin sensitivity support a central role for the liver in mediating the effects of physical activity in 


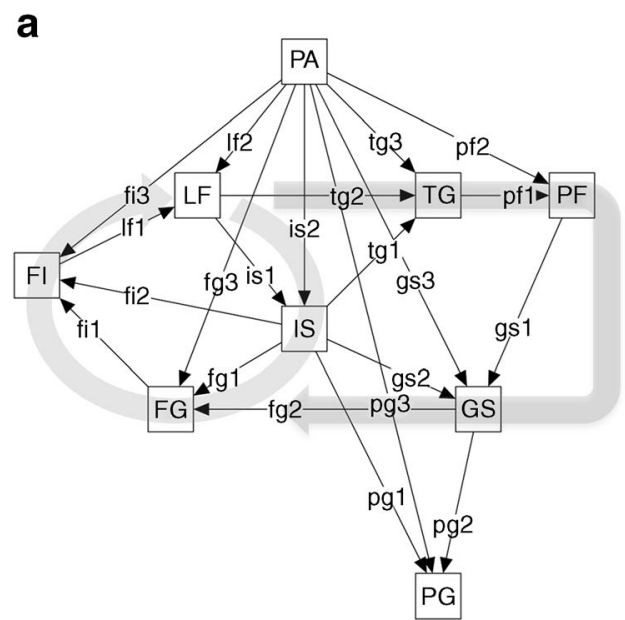

c

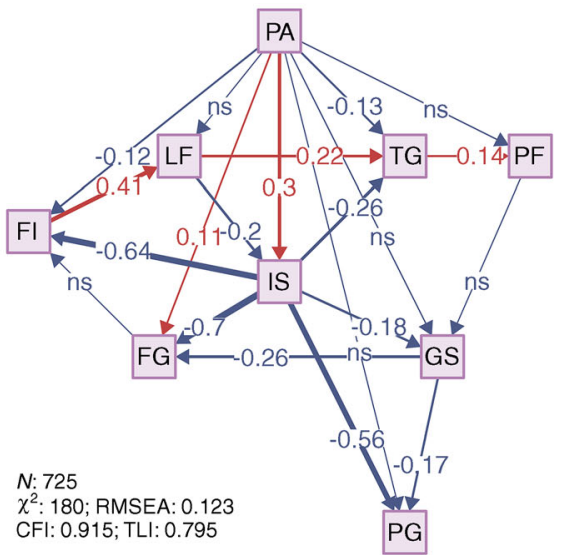

Fig. 2 TC-PA structural equation model definition diagram, fit estimates and effect estimate diagrams from a hypothesised model for the role of physical activity and liver fat in glycaemic control. (a) Model definitions, with squares representing manifest nodes and arrows indicating regression coefficients pointing towards an outcome of a respective regression. (b) Model fit; density plot of model fit $\chi^{2}$ from variablerandomised comparable structural equation models applied on respective dataset (10,000 iterations). Dashed vertical lines indicate TC-PA model

glycaemic control (see Fig. 1 and Table 2). Specifically, the $\mathrm{PA} \rightarrow \mathrm{IS} \uparrow, \mathrm{IS} \rightarrow \mathrm{FI} \downarrow, \mathrm{FI} \rightarrow \mathrm{LF} \uparrow, \mathrm{IS} \rightarrow \mathrm{FG} \downarrow$ and $\mathrm{IS} \rightarrow \mathrm{PG} \downarrow$ associations were statistically robust within and between cohorts. Indeed, pathways $\mathrm{PA} \rightarrow \mathrm{IS} \uparrow \rightarrow \mathrm{FG} \downarrow$ and $\mathrm{PA} \rightarrow \mathrm{IS} \uparrow \rightarrow \mathrm{PG} \downarrow$ are consistent in direction and magnitude in both cohorts, providing reassurance that these findings are not false positives, and suggesting that physical activity exerts effects on glucose homeostasis via insulin sensitivity in a similar way before and after the onset of type 2 diabetes. The pathways PA $\rightarrow$ $\mathrm{FI} \downarrow \rightarrow \mathrm{LF} \downarrow \rightarrow \mathrm{IS} \downarrow \rightarrow \mathrm{FG} \downarrow$ and $\mathrm{PA} \rightarrow \mathrm{FI} \downarrow \rightarrow \mathrm{LF} \downarrow \rightarrow \mathrm{IS} \uparrow \rightarrow \mathrm{PG} \downarrow$ were only tested in cohort 1 , as the direct effect estimate $\mathrm{LF} \rightarrow \mathrm{IS}$ was not statistically significant in cohort 2 .

A core feature of the TC relates to the role of islet triacylglycerol content in beta cell lipotoxicity, leading to diminished beta cell function [16]. As beta cells are estimated to account for

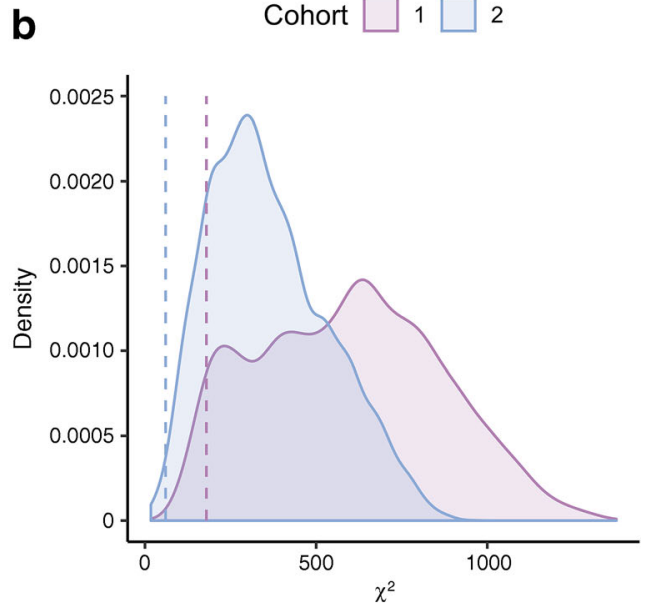

d

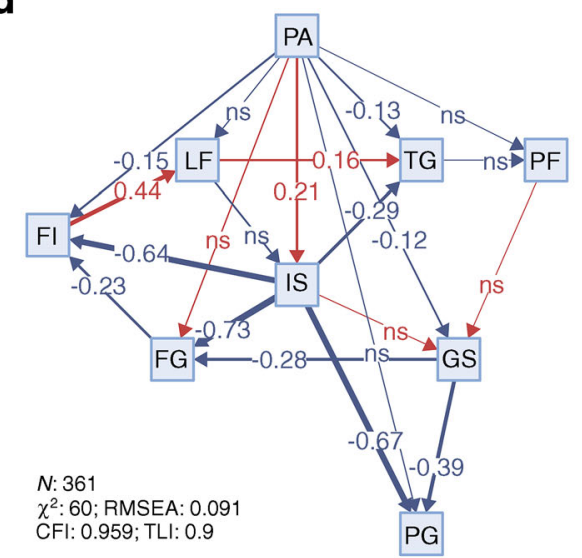

$\chi^{2}$, solid lines and shaded areas indicate $\chi^{2}$ of all null iterations. $(\mathbf{c}, \mathbf{d})$ Effect estimate diagrams of the defined model applied on cohort 1 (no diabetes/prediabetes, $\mathbf{c}$ ) and cohort 2 (type 2 diabetes, $\mathbf{d}$ ), where the arrow thickness is weighted by effect estimate magnitude, and colours red and blue indicate positive and negative estimates, respectively. All continuous variables are normally transformed and adjusted for: age, sex, metformin treatment (cohort 2), study centre, total energy intake, and carbohydrate, fat and protein intake. See Text box for node and edge abbreviations

only a small fraction of total pancreatic volume $(<5 \%$ [37]), MRI imaging of the pancreas may be too insensitive to specifically quantify beta cell triacylglycerol content, with the signal instead being driven by whole pancreas fat content [38]. Furthermore, the serrations and involutions that characterise some pancreases [39] make the accurate assessment of ectopic fat near the boundary of the organ challenging, even using whole-organ MRI techniques and experienced radiographers identifying organ boundaries, as was the case here. The shape of the pancreas may also influence (or be correlated with factors that influence) diabetes remission following very-low-energy diets, such that improvements in early insulin secretion are greater in individuals with regularly rather than irregularly shaped pancreases [40]. However, there is a clear possibility that if pancreas shape and pancreatic fat measurement error 
Table 2 Individual edge effect estimates for the TC and TC-PA structural equation models

Outcome node/parent node (edge)
Cohort 1

(no diabetes/prediabetes)

$\beta \quad \beta \mathrm{SE} \quad p$ value

TC model

OGIS/

LF (is1)

LF/

FI (lf1)

FI/

FG (fi1)

IS (fi2)

FG/

IS (fg1)

GS (fg2)

TG/

IS (tg1)

LF (tg2)

$\mathrm{PF} /$

$$
\text { TG (pfl) }
$$

GS/

$\mathrm{PF}$ (gs1)

IS (gs2)

PG/

IS (pg1)

GS (pg2)

TC-PA model

IS/

$$
\text { PA (is2) }
$$

LF (is1)

LF/

PA (lf2)

FI (lfl)

FI/

PA (fi3)

FG (fil)

IS (fi2)

FG/

PA (fg3)

IS (fg1)

GS (fg2)

TG/

PA (tg3)

OGIS (tg1)

LF (tg2)

$\mathrm{PF} /$

$$
\text { PA (pf2) }
$$

TG (pfl)

GS/

PA (gs3)

PF (gs1)
$-0.23$

0.41

$-0.06$

$-0.70$

$-0.67$

$-0.26$

$-0.31$

0.22

0.14

$-0.05$

$-0.21$

$-0.58$

$-0.17$

0.30

$-0.20$

$-0.04$

0.41

$-0.12$

$-0.04$

$-0.64$

0.11

$-0.70$

$-0.26$

$-0.13$

$-0.26$

0.22

$-0.06$

0.14

$-0.05$

$-0.05$
0.04

0.04

0.03

0.03

0.03

0.03

0.03

0.03

0.03

0.04

0.03

0.03

0.03

0.04

0.04

0.04

0.04

0.03

0.03

0.04

0.03

0.03

0.03

0.04

0.04

0.04

0.03

0.03

0.04

0.05
$<0.001$

$<0.001$

0.05

$<0.001$

$<0.001$

$<0.001$

$<0.001$

$<0.001$

$<0.001$

0.185

$<0.001$

$<0.001$

$<0.001$

$<0.001$

$<0.001$

0.234

$<0.001$

$<0.001$

0.265

$<0.001$

0.001

$<0.001$

$<0.001$

$<0.001$

$<0.001$

$<0.001$

0.067

$<0.001$

0.212

0.314
Cohort 2 (diabetes)

$\bar{\beta} \quad \beta$ SE $\quad p$ value

0.44

0.06

0.09

$<0.001$

$-0.25$

0.05

$<0.001$

$-0.70$

0.06

$<0.001$

$-0.73$

0.06

$<0.001$

$-0.31$

0.03

0.03

$<0.001$

$-0.34$

0.05

0.05

$<0.001$

0.13

0.01

0.05

0.886

$<0.01$

0.05

0.967

0.02

0.05

0.63

$-0.67$

0.03

$<0.001$

$-0.38$

0.03

$<0.001$

0.21

0.05

0.07

$<0.001$

$-0.12$

0.065

$-0.04$

0.05

0.447

0.44

0.05

$<0.001$

$-0.16$

0.05

0.001

$-0.23$

0.07

$<0.001$

$-0.64$

0.07

$<0.001$

0.06

0.03

0.061

$-0.73$

0.03

$<0.001$

$-0.28$

0.03

$<0.001$

$-0.13$

0.05

0.012

$-0.30$

0.05

$<0.001$

0.16

0.05

0.002

$-0.01$

0.06

0.809

$-0.01$

0.05

0.819

$-0.12$

0.06

0.032

0.01

0.05 
Table 2 (continued)

\begin{tabular}{|c|c|c|c|c|c|c|}
\hline \multirow[t]{2}{*}{$\begin{array}{l}\text { Outcome node/parent } \\
\text { node (edge) }\end{array}$} & \multicolumn{3}{|c|}{$\begin{array}{l}\text { Cohort } 1 \\
\text { (no diabetes/prediabetes) }\end{array}$} & \multicolumn{3}{|c|}{ Cohort 2 (diabetes) } \\
\hline & $\beta$ & $\beta \mathrm{SE}$ & $p$ value & $\beta$ & $\beta \mathrm{SE}$ & $p$ value \\
\hline IS (gs2) & -0.18 & 0.04 & $<0.001$ & 0.02 & 0.05 & 0.696 \\
\hline \multicolumn{7}{|l|}{$\mathrm{PG} /$} \\
\hline PA (pg3) & -0.04 & 0.03 & 0.194 & -0.05 & 0.04 & 0.162 \\
\hline IS (pg1) & -0.56 & 0.03 & $<0.001$ & -0.67 & 0.03 & $<0.001$ \\
\hline GS (pg2) & -0.17 & 0.03 & $<0.001$ & -0.39 & 0.03 & $<0.001$ \\
\hline
\end{tabular}

All continuous variables were normally transformed and adjusted for age, sex, metformin treatment (cohort 2), study centre, total energy intake and carbohydrate, fat and protein intake

See Text box for node and edge abbreviations

correlate, the apparent relationship of pancreas fat and beta cell function may not be causal. Notwithstanding the difficulty in assessing pancreatic fat, we do observe pairwise correlations between pancreatic fat and the other metabolic outcomes in cohort 1 (see ESM Fig. 1), consistent with observations made by Tushuizen et al [41] who noted a relationship between pancreatic fat and beta cell function in prediabetes but not type 2 diabetes. Thus, pancreatic fat may have a significant role in glucose homeostasis, though we could not determine in our analyses if this is mediated through beta cell function.

As anticipated, we also observed differences in the association of insulin sensitivity with beta cell function between the prediabetes and the diabetes cohorts. In the prediabetes cohort, beta cell function and insulin sensitivity were related; this was not the case in the diabetes cohort, probably because the capacity to compensate for peripheral insulin resistance by secreting more insulin is greater in prediabetes than once diabetes is manifest [42].
Our findings should also be considered in light of those from the 'Primary care-led weight management for remission of type 2 diabetes trial' (DiRECT) in which the remission of type 2 diabetes in response to an extended period of weight management through restriction of energy intake was studied [43] (note that DiRECT is a different study to IMI DIRECT). The results from a follow-up study to DiRECT, where a subgroup of participants $(n=88)$ underwent more detailed physiological testing similar to those in the present study, is of particular importance [44]. In this study neither liver fat nor pancreatic fat differed statistically between responders (those who remained free from type 2 diabetes after 12 months) and non-responders, though it did decrease in both groups in response to the intervention compared with the control arm. Moreover, first-phase insulin secretion improved in the responders whereas no difference was observed in the nonresponders. The implication of this is that a reduction in both liver and pancreatic fat may coincide with remission of type 2 diabetes, and even be necessary, but it is not sufficient (as non-
Table 3 Pathway (mediation) effect estimates for the association of physical activity with glycaemic control within the TCPA model (see Fig. 2)

\begin{tabular}{|c|c|c|c|c|c|c|c|}
\hline \multirow[b]{2}{*}{ Outcome node } & \multirow[b]{2}{*}{ Edge path } & \multicolumn{3}{|c|}{ Cohort 1 (no diabetes/prediabetes) } & \multicolumn{3}{|c|}{ Cohort 2 (diabetes) } \\
\hline & & $\beta$ & $\beta \mathrm{SE}$ & $p$ value & $\beta$ & $\beta \mathrm{SE}$ & $p$ value \\
\hline FG & $\mathrm{PA} \rightarrow \mathrm{IS} \rightarrow \mathrm{FG}$ & -0.212 & 0.026 & $<0.001$ & -0.153 & 0.039 & $<0.001$ \\
\hline PG & $\mathrm{PA} \rightarrow \mathrm{IS} \rightarrow \mathrm{PG}$ & -0.171 & 0.022 & $<0.001$ & -0.140 & 0.036 & $<0.001$ \\
\hline FG & $\mathrm{PA} \rightarrow \mathrm{IS} \rightarrow \mathrm{GS} \rightarrow \mathrm{FG}$ & 0.015 & 0.004 & $<0.001$ & & & \\
\hline PG & $\mathrm{PA} \rightarrow \mathrm{IS} \rightarrow \mathrm{GS} \rightarrow \mathrm{PG}$ & 0.009 & 0.003 & 0.001 & & & \\
\hline FG & $\mathrm{PA} \rightarrow \mathrm{FI} \rightarrow \mathrm{LF} \rightarrow \mathrm{IS} \rightarrow \mathrm{FG}$ & -0.007 & 0.002 & 0.002 & & & \\
\hline PG & $\mathrm{PA} \rightarrow \mathrm{FI} \rightarrow \mathrm{LF} \rightarrow \mathrm{IS} \rightarrow \mathrm{PG}$ & -0.006 & 0.002 & 0.002 & & & \\
\hline FG & $\mathrm{PA} \rightarrow \mathrm{GS} \rightarrow \mathrm{FG}$ & & & & 0.033 & 0.016 & 0.037 \\
\hline PG & $\mathrm{PA} \rightarrow \mathrm{GS} \rightarrow \mathrm{PG}$ & & & & 0.045 & 0.022 & 0.04 \\
\hline
\end{tabular}

All continuous variables are normally transformed and adjusted for age, sex, metformin treatment (cohort 2), study centre, total energy intake and carbohydrate, fat and protein intake

See Text box for node abbreviations 
responders also had a decrease in both). The lack of a relationship between pancreatic fat and glucose sensitivity in our study could be for the same reason as in DiRECT.

A weakness of this analysis is that it is cross-sectional and, thus, the direction of the effects between some variables in the model cannot be easily ascertained. Nevertheless, the effects tested here and the structure of the models were prespecified, based on a biologically plausible hypothesis proposed previously [45]. As we have sought to test specifically the TC here we have also limited the model to edges (and directions) reflecting this hypothesis only. For this reason the model does not include some commonly hypothesised direct effect edges such as beta cell glucotoxicity and lipotoxicity (not mediated by pancreatic fat) [46], the rate sensitivity and potentiation fraction ratio variables in beta cell function modelled from oral glucose tolerance tests (often included alongside glucose sensitivity) [22].

Another consideration is the mathematical relatedness of fasting insulin, fasting glucose and $2 \mathrm{~h}$ glucose with insulin sensitivity, which are determined using some of the same variables derived from the fsOGTT/MMTT and are thus implicitly correlated. Nevertheless, the OGIS method has been extensively validated and the OGIS estimate has shown to be a close representation of the gold-standard $M$ value from a euglycaemic-hyperinsulinaemic clamp [47]. As such, it is also worth bearing in mind that OGIS, as a whole-body insulin sensitivity measure, will not only reflect hepatic insulin sensitivity but also skeletal muscle insulin sensitivity. This is particularly important for the results of this study where the effects of physical activity on glycaemic control mediated through insulin sensitivity likely reflect an insulin-sensitising effect on skeletal muscle and not only the liver.

It is possible that the methods used here to normalise data distributions and control for confounding reduce statistical power to detect effects. Despite this caveat, the approach is valuable, as it renders the effects estimated within and between the two cohorts directly comparable, minimising confounding and bias, while also restricting the parameterisation of the model. An important limitation of any multivariable analysis, where 'conditioning' variables (covariates) are on the causal pathway between the defined exposures and outcomes, is that effects will be underestimated owing to mediation $[30,48]$. In the current analysis, this is unlikely to be a cause for concern, as physical activity, for example, does not determine sex or chronological age; thus, neither sex nor age can mediate the effects of physical activity on metabolic outcomes.

Conclusion This analysis highlights that peripheral insulin sensitivity and liver fat are likely to be major mediators of the effects of physical activity in whole-body glucose homeostasis, key features of the pathogenic cycle proposed by Taylor $[15,16]$. This study illustrates the value of large well-phenotyped cohorts, where emphasis is placed on detailed phenotypic assessments that allow structures of physiological relationships to be modelled and complex multi-dimensional pathogenic processes to be assessed.

Acknowledgements Open access funding provided by Lund University. We thank all the participants in IMI DIRECT for their contribution to the study. More information on IMI DIRECT is available on http://www. direct-diabetes.org/.

Data availability Requests for access to IMI DIRECT data, including data presented here, can made to DIRECTdataaccess@ Dundee.ac.uk.

Funding The work leading to this publication has received support from the Innovative Medicines Initiative Joint Undertaking under grant agreement no. 115317 (DIRECT), resources of which are composed of financial contribution from the European Union's Seventh Framework Programme (FP7/2007-2013) and EFPIA companies in kind contribution. RWK was funded by a STAR Novo Nordisk co-financed $\mathrm{PhD}$ fellowship and a Novo Nordisk Foundation (NNF18OC0031650) postdoctoral fellowship. TW was funded by a PhD fellowship from MedImmune. SBra was funded by the UK Medical Research Council (MC_UU_12015/3). This study was undertaken as part of a research programme supported by the European Research Council (CoG-2015 681742_NASCENT). IMI DIRECT has received funding from EFPIA members, including Boehringer Ingelheim, Eli Lilly, Novo Nordisk, Servier Laboratories and Sanofi-Aventis. No study funders were involved in the design of the study; the collection, analysis, and interpretation of data; writing the report; or the decision to submit the report for publication.

Duality of interest IP is an employee of Eli Lilly GmbH, Vienna, Austria. $\mathrm{HR}$ is an employee of Sanofi-Aventis Deutschland GmbH, Frankfurt am Main, Germany. This does not alter our adherence to Diabetologia's policies on sharing data and materials. The views expressed in this article are those of the author(s) and not necessarily those of the NHS, the NIHR or the Department of Health. MIMcC has served on advisory panels for Pfizer, Novo Nordisk and Zoe Global, received honoraria from Merck, Pfizer, Novo Nordisk and Eli Lilly and research funding from AbbVie, AstraZeneca, Boehringer Ingelheim, Eli Lilly, Janssen, Merck, Novo Nordisk, Pfizer, Roche, Sanofi-Aventis, Servier and Takeda. As of June 2019, MIMcC is an employee of Genentech, and a holder of Roche stock. All other authors declare that there is no duality of interest associated with their contribution to this manuscript.

Contribution statement RWK wrote the manuscript and carried out the statistical analyses presented here. RWK conceptualised and designed the analysis with contributions from PWF, NA-P, GNG, AK, AMah and MIMcC. PWF, MW and EP conceptualised and designed the cohorts with contributions from RWK, JA, JBel, JBeu., SBru, ETD, GF, TH, AH, ML, AMar, TJMcD, OP, JMS, HJAT, AMah, MIMcC, HR, MW, EP and IP. PWF, RWK, GNG, TW, JBel, JBeu, SBra, FDe M, IMF, GF, THH, TK, AK, AMar, TJMcD, FR, ELT, AV and AMah contributed to sample assaying, data analysis/processing and/or data quality control procedures. RWK, GNG, IMF, THH, TH, AH, TK, ML, AMar, TJMcD, OP, FR, JBeu, MW, EP and PWF contributed to data collection at study centres. All authors contributed to drafting the article and/or revising it critically for important intellectual content. All authors approved the final version of the manuscript. All authors accept responsibility for all aspects of the work insofar as ensuring that questions related to the accuracy or integrity of any part of the article are appropriately investigated and resolved. RWK is the guarantor of this work and, as such, had full access to the data analysed here and takes responsibility for the integrity of the data and its analysis. 
Open Access This article is licensed under a Creative Commons Attribution 4.0 International License, which permits use, sharing, adaptation, distribution and reproduction in any medium or format, as long as you give appropriate credit to the original author(s) and the source, provide a link to the Creative Commons licence, and indicate if changes were made. The images or other third party material in this article are included in the article's Creative Commons licence, unless indicated otherwise in a credit line to the material. If material is not included in the article's Creative Commons licence and your intended use is not permitted by statutory regulation or exceeds the permitted use, you will need to obtain permission directly from the copyright holder. To view a copy of this licence, visit http://creativecommons.org/licenses/by/4.0/.

\section{References}

1. International DiabetesFederation (2015) IDF diabetes atlas, 7 th edn. IDF, Brussels

2. NCD Risk Factor Collaboration (NCD-RisC) (2016) Trends in adult body-mass index in 200 countries from 1975 to 2014: a pooled analysis of 1698 population-based measurement studies with 19.2 million participants. Lancet 387(10026):1377-1396. https://doi.org/10.1016/s0140-6736(16)30054-x

3. Sallis JF, Bull F, Guthold R et al (2016) Progress in physical activity over the Olympic quadrennium. Lancet 388(10051):1325-1336. https://doi.org/10.1016/S0140-6736(16)30581-5

4. Colberg SR, Sigal RJ, Fernhall B et al (2010) Exercise and type 2 diabetes: the American College of Sports Medicine and the American Diabetes Association: joint position statement. Diabetes Care 33(12):e147-e167. https://doi.org/10.2337/dc10-9990

5. Poveda A, Koivula RW, Ahmad S et al (2016) Innate biology versus lifestyle behaviour in the aetiology of obesity and type 2 diabetes: the GLACIER study. Diabetologia 59(3):462-471. https://doi.org/10.1007/s00125-015-3818-y

6. Lahjibi E, Heude B, Dekker JM et al (2013) Impact of objectively measured sedentary behaviour on changes in insulin resistance and secretion over 3 years in the RISC study: interaction with weight gain. Diabetes Metab 39(3):217-225. https://doi.org/10.1016/j. diabet.2012.12.006

7. Philipsen A, Hansen AL, Jorgensen ME et al (2015) Associations of objectively measured physical activity and abdominal fat distribution. Med Sci Sports Exerc 47(5):983-989. https://doi.org/10. 1249/mss.0000000000000504

8. Henson J, Edwardson CL, Morgan B et al (2015) Associations of sedentary time with fat distribution in a high-risk population. Med Sci Sports Exerc 47(8):1727-1734. https://doi.org/10.1249/mss. 0000000000000572

9. Gastaldelli A, Kozakova M, Hojlund K et al (2009) Fatty liver is associated with insulin resistance, risk of coronary heart disease, and early atherosclerosis in a large European population. Hepatology 49(5):1537-1544. https://doi.org/10.1002/hep.22845

10. Kotronen A, Seppala-Lindroos A, Bergholm R, Yki-Jarvinen H (2008) Tissue specificity of insulin resistance in humans: fat in the liver rather than muscle is associated with features of the metabolic syndrome. Diabetologia 51(1):130-138. https://doi.org/10. 1007/s00125-007-0867-x

11. Kotronen A, Juurinen L, Tiikkainen M, Vehkavaara S, Yki-Jarvinen $\mathrm{H}$ (2008) Increased liver fat, impaired insulin clearance, and hepatic and adipose tissue insulin resistance in type 2 diabetes. Gastroenterology 135(1):122-130. https://doi.org/10.1053/j.gastro. 2008.03.021

12. De Silva NMG, Borges MC, Hingorani A et al (2019) Liver function and risk of type 2 diabetes: bidirectional mendelian randomization study. Diabetes 68(8):1681-1691. https://doi.org/ $10.2337 / \mathrm{db} 18-1048$

13. Liu Z, Zhang Y, Graham S, et al (2019) Mendelian randomization analysis dissects the relationship between NAFLD, T2D, and obesity and provides implications to precision medicine. bioRxiv: 657734. https://doi.org/10.1101/657734

14. Reaven GM (1988) Role of insulin resistance in human disease. Diabetes 37(12):1595-1607. https://doi.org/10.2337/diab.37.12. 1595

15. Taylor R (2008) Pathogenesis of type 2 diabetes: tracing the reverse route from cure to cause. Diabetologia 51(10):1781-1789. https:// doi.org/10.1007/s00125-008-1116-7

16. Taylor R (2013) Type 2 diabetes: etiology and reversibility. Diabetes Care 36(4):1047-1055. https://doi.org/10.2337/dc121805

17. Koivula RW, Heggie A, Barnett A et al (2014) Discovery of biomarkers for glycaemic deterioration before and after the onset of type 2 diabetes: rationale and design of the epidemiological studies within the IMI DIRECT Consortium. Diabetologia 57(6): 1132-1142. https://doi.org/10.1007/s00125-014-3216-x

18. Koivula RW, Forgie IM, Kurbasic A et al (2019) Discovery of biomarkers for glycaemic deterioration before and after the onset of type 2 diabetes: descriptive characteristics of the epidemiological studies within the IMI DIRECT Consortium. Diabetologia 62(9): 1601-1615. https://doi.org/10.1007/s00125-019-4906-1

19. ADA (2018) Classification and diagnosis of diabetes: standards of medical care in diabetes-2018. Diabetes Care 41(Suppl 1):S13S27. https://doi.org/10.2337/dc18-S002

20. Rauh SP, Heymans MW, Koopman AD et al (2017) Predicting glycated hemoglobin levels in the non-diabetic general population: development and validation of the DIRECT-DETECT prediction model: a DIRECT study. PLoS One 12(2):e0171816. https://doi. org/10.1371/journal.pone.0171816

21. Mari A, Pacini G, Murphy E, Ludvik B, Nolan JJ (2001) A modelbased method for assessing insulin sensitivity from the oral glucose tolerance test. Diabetes Care 24(3):539-548. https://doi.org/10. 2337/diacare.24.3.539

22. Mari A, Ferrannini E (2008) $\beta$-cell function assessment from modelling of oral tests: an effective approach. Diabetes Obes Metab 10(Suppl 4):77-87. https://doi.org/10.1111/j.1463-1326. 2008.00946.x

23. Thomas EL, Fitzpatrick JA, Malik SJ, Taylor-Robinson SD, Bell JD (2013) Whole body fat: content and distribution. Prog Nucl Magn Reson Spectrosc 73:56-80. https://doi.org/10.1016/j.pnmrs. 2013.04.001

24. O'Regan DP, Callaghan MF, Wylezinska-Arridge M et al (2008) Liver fat content and $\mathrm{T} 2 *$ : simultaneous measurement by using breath-hold multiecho MR imaging at 3.0 T-feasibility. Radiology 247(2):550-557. https://doi.org/10.1148/radiol. 2472070880

25. White T, Westgate K, Wareham NJ, Brage S (2016) Estimation of physical activity energy expenditure during free-living from wrist accelerometry in UK adults. PLoS One 11(12):e0167472. https:// doi.org/10.1371/journal.pone.0167472

26. White T, Westgate K, Hollidge $\mathrm{S}$ et al (2019) Estimating energy expenditure from wrist and thigh accelerometry in free-living adults: a doubly labelled water study. Int J Obes 43(11):23332342. https://doi.org/10.1038/s41366-019-0352-x

27. Brage S, Westgate K, Wijndaele K, Godinho J, Griffin S, Wareham N (2013) Evaluation of a method for minimising diurnal information bias in objective sensor data. Int Conf Amb Mon Phys Act Mov

28. Sobel ME (1982) Asymptotic confidence intervals for indirect effects in structural equation models. Sociol Methodol 13:290 312. https://doi.org/10.2307/270723

29. Baron RM, Kenny DA (1986) The moderator-mediator variable distinction in social psychological research: conceptual, strategic, 
and statistical considerations. J Pers Soc Psychol 51(6):1173-1182. https://doi.org/10.1037//0022-3514.51.6.1173

30. West SG, Taylor AB, Wu W (2012) Model fit and model selection in structural equation modeling. The Guilford Press, New York

31. Bentler PM (1990) Comparative fit indexes in structural models. Psychol Bull 107(2):238-246. https://doi.org/10.1037/0033-2909. 107.2.238

32. Tucker LR, Lewis C (1973) A reliability coefficient for maximum likelihood factor analysis. Psychometrika 38(1):1-10. https://doi. org/10.1007/bf02291170

33. Bentler PM (1995) EQS structural equations program manual. Multivariate Software, Encino

34. Steiger JH, Lind JC (1980) Statistically based tests for the number of common factors. In: Annual meeting of the Psychometric Society, Iowa City. Vol 758, pp 424-453

35. R-Core-Team (2014) R: A language and environment for statistical computing. R Foundation for Statistical Computing, Vienna

36. Rosseel Y (2012) lavaan: an R package for structural equation modeling. J Stat Softw 48(2). https://doi.org/10.18637/jss.v048.i02

37. Brissova M, Fowler MJ, Nicholson WE et al (2005) Assessment of human pancreatic islet architecture and composition by laser scanning confocal microscopy. J Histochem Cytochem 53(9):10871097. https://doi.org/10.1369/jhc.5C6684.2005

38. Al-Mrabeh A, Hollingsworth KG, Steven S, Tiniakos D, Taylor R (2017) Quantification of intrapancreatic fat in type 2 diabetes by MRI. PLoS One 12(4):e0174660. https://doi.org/10.1371/journal. pone. 0174660

39. Macauley M, Percival K, Thelwall PE, Hollingsworth KG, Taylor R (2015) Altered volume, morphology and composition of the pancreas in type 2 diabetes. PLoS One 10(5):e0126825. https:// doi.org/10.1371/journal.pone.0126825

40. Al-Mrabeh A, Hollingsworth KG, Steven S, Taylor R (2016) Morphology of the pancreas in type 2 diabetes: effect of weight loss with or without normalisation of insulin secretory capacity. Diabetologia 59(8):1753-1759. https://doi.org/10.1007/s00125016-3984-6
41. Tushuizen ME, Bunck MC, Pouwels PJ et al (2007) Pancreatic fat content and $\beta$-cell function in men with and without type 2 diabetes. Diabetes Care 30(11):2916-2921. https://doi.org/10.2337/ dc07-0326

42. Defronzo RA (2009) Banting lecture. From the triumvirate to the ominous octet: a new paradigm for the treatment of type 2 diabetes mellitus. Diabetes 58(4):773-795. https://doi.org/10.2337/db099028

43. Lean MEJ, Leslie WS, Barnes AC et al (2018) Primary care-led weight management for remission of type 2 diabetes (DiRECT): an open-label, cluster-randomised trial. Lancet 391(10120):541-551. https://doi.org/10.1016/S0140-6736(17)33102-1

44. Taylor R, Al-Mrabeh A, Zhyzhneuskaya S et al (2018) Remission of human type 2 diabetes requires decrease in liver and pancreas fat content but is dependent upon capacity for $\beta$ cell recovery. Cell Metab 28(4):547-556.e543. https://doi.org/10.1016/j.cmet.2018. 07.003

45. Kline RB (2012) Assumptions in structural equation modeling. In: Hoyle RH (ed) Handbook of structural equation modeling. The Guilford Press, New York, pp 111-125

46. Del Prato S (2009) Role of glucotoxicity and lipotoxicity in the pathophysiology of type 2 diabetes mellitus and emerging treatment strategies. Diabet Med 26(12):1185-1192. https://doi.org/10.1111/j. 1464-5491.2009.02847.x

47. Mari A, Pacini G, Brazzale AR, Ahrén B (2005) Comparative evaluation of simple insulin sensitivity methods based on the oral glucose tolerance test. Diabetologia 48(4):748-751. https://doi. org/10.1007/s00125-005-1683-9

48. Demissie S, Cupples LA (2011) Bias due to two-stage residualoutcome regression analysis in genetic association studies. Genet Epidemiol 35(7):592-596. https://doi.org/10.1002/gepi.20607

Publisher's note Springer Nature remains neutral with regard to jurisdictional claims in published maps and institutional affiliations. 


\section{Affiliations}

Robert W. Koivula ${ }^{1,2}$ (D) Naeimeh Atabaki-Pasdar ${ }^{1} \cdot$ Giuseppe N. Giordano $^{1} \cdot$ Tom White $^{3}$ • Jerzy Adamski ${ }^{4,5,6}$.

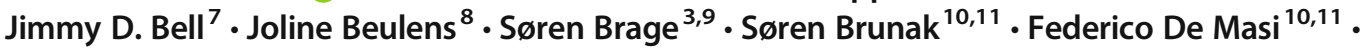
Emmanouil T. Dermitzakis ${ }^{12,13,14} \cdot \operatorname{lan}$ M. Forgie ${ }^{15} \cdot$ Gary Frost $^{16} \cdot$ Torben Hansen $^{9,17} \cdot$ Tue H. Hansen $^{17}$. Andrew Hattersley ${ }^{18,19}$ - Tarja Kokkola ${ }^{20}$ - Azra Kurbasic ${ }^{1}$ - Markku Laakso ${ }^{20}$. Andrea Mari ${ }^{21} \cdot$ Timothy J. McDonald $^{18}$. Oluf Pedersen $^{17}$. Femke Rutters ${ }^{8}$ • Jochen M. Schwenk ${ }^{22} \cdot$ Harriet J. A. Teare ${ }^{23}$. E. Louise Thomas ${ }^{7}$.

Ana Vinuela ${ }^{12,13,14}$ - Anubha Mahajan ${ }^{24}$ - Mark I. McCarthy 2,24,25,26 Hartmut Ruetten $^{27}$ - Mark Walker $^{28}$. Ewan Pearson $^{15} \cdot$ Imre Pavo $^{29}$ - Paul W. Franks ${ }^{1,2,30,31} \cdot$ for the IMI DIRECT Consortium

1 Department of Clinical Sciences, Lund University, Genetic and Molecular Epidemiology, CRC, Skåne University Hospital Malmö, Building 91, Level 12, Jan Waldenströms gata 35, SE-205 02 Malmö, Sweden

2 Oxford Centre for Diabetes, Endocrinology and Metabolism, Radcliffe Department of Medicine, University of Oxford, Oxford, UK

3 MRC Epidemiology Unit, University of Cambridge School of Clinical Medicine, Cambridge, UK

4 Research Unit Molecular Endocrinology and Metabolism, Genome Analysis Center, Helmholtz Zentrum München, German Research Center for Environmental Health, Neuherberg, Germany

5 Lehrstuhl für Experimentelle Genetik, Technische Universität München, Freising-Weihenstephan, Germany

6 Department of Biochemistry, Yong Loo Lin School of Medicine, National University of Singapore, Singapore, Republic of Singapore

7 Research Centre for Optimal Health, Department of Life Sciences, University of Westminister, London, UK

8 Department of Epidemiology and Biostatistics, Amsterdam Public Health Research Institute, Amsterdam University Medical Centre, location VU University Medical Center, Amsterdam, the Netherlands

9 Faculty of Health Sciences, University of Southern Denmark, Odense, Denmark

10 The Novo Nordisk Foundation Center for Protein Research, University of Copenhagen, Copenhagen, Denmark

11 Department of Bio and Health Informatics, Technical University of Denmark, Lyngby, Denmark

12 Department of Genetic Medicine and Development, University of Geneva Medical School, Geneva, Switzerland

13 Institute of Genetics and Genomics in Geneva (iGE3), University of Geneva, Geneva, Switzerland

14 Swiss Institute of Bioinformatics, Geneva, Switzerland

15 Population Health \& Genomics, School of Medicine, University of Dundee, Ninewells Hospital, Dundee, UK
16 Nutrition and Dietetics Research Group, Department of Medicine, Division of Diabetes, Endocrinology and Metabolism, Imperial College London, Hammersmith Campus, London, UK

17 The Novo Nordisk Foundation Center for Basic Metabolic Research, Faculty of Health and Medical Science, University of Copenhagen, Copenhagen, Denmark

18 NIHR Exeter Clinical Research Facility, University of Exeter Medical School, Exeter, UK

19 Institute of Biomedical and Clinical Science, University of Exeter Medical School, Exeter, UK

20 Department of Medicine, University of Eastern Finland and Kuopio University Hospital, Kuopio, Finland

21 Institute of Neurosciences, National Research Council, Padova, Italy

22 Affinity Proteomics, Science for Life Laboratory, KTH - Royal Institute of Technology, Stockholm, Sweden

23 HeLEX, Nuffield Department of Population Health, University of Oxford, Old Road Campus, Headington, Oxford, UK

24 Wellcome Centre for Human Genetics, University of Oxford, Oxford, UK

25 NIHR Oxford Biomedical Research Centre, Churchill Hospital, Oxford, UK

26 Human Genetics, Genentech, South San Francisco, CA, USA

27 Sanofi-Aventis Deutschland GmbH, R\&D, Frankfurt am Main, Germany

28 Institute of Cellular Medicine (Diabetes), Newcastle University, Newcastle upon Tyne, UK

29 Eli Lilly Regional Operations GmbH, Vienna, Austria

30 Department of Nutrition, Harvard School of Public Health, Boston, MA, USA

31 Department of Public Health \& Clinical Medicine, Section for Medicine, Umeå University Hospital, Umeå, Sweden 